\title{
PIME Aprendizaje Basado en Proyectos: Ingeniería de Carreteras e Ingeniería Geotécnica.
}

M.E. Garrido de la Torre ${ }^{a}$, A. M. Pérez-Zuriagab ${ }^{b}$ V. Martínez-Ibáñez ${ }^{c}$, G. López Maldonado ${ }^{d}$ y A. Cuadrado Tarodo ${ }^{\mathrm{e}}$

a Departamento Ingeniería del Terreno, Universitat Politècnica de València. Email: egarrido@trr.upv.es; ${ }^{\mathrm{b}}$ Departamento Ingeniería e Infraestructura de los Transportes, Universitat Politècnica de València. Email: anpezu@tra.upv.es; ${ }^{c}$ Departamento Ingeniería del Terreno, Universitat Politècnica de València. Email vicmarib@trr.upv.es; ${ }^{d}$ Departamento Ingeniería e Infraestructura de los Transportes, Universitat Politècnica de València. Email: grilomal@tra.upv.es; ${ }^{e}$ Departamento Ingeniería e Infraestructura de los Transportes, Universitat Politècnica de València. Email: alcuata@tra.upv.es.

\begin{abstract}
In recent years, the Universitat Politècnica de València has encouraged the implementation of Educational Innovation and Improvement Projects (PIME) within the "Learning and Teaching" $(A+D)$ program. During the 2019/2020 academic year, a PIME based on Problem or Project Based Learning (PBL) was implemented, bringing together two consecutive subjects from the third year of Civil Engineering. This article shows the results achieved in the first semester after implantation. The students have clearly expressed their interest and preference for this kind of methodology. They have obtained an in-depth learning and they are prepared to acquire knowledge by themselves, as well as transferring it to professional practice by developing their ability to work as a team and the possibility of visit the studio area, so the knowledges acquire a practical character. They have also developed the expected skills, integrating academic aspects with other social and ethical ones.
\end{abstract}

Keywords: PIME, PBL, specific skills, transversal skills, civil engineering, active teaching, geotechnical engineering, highway engineering

\section{Resumen}

En los últimos años, la Universitat Politècnica de València (UPV) ha promovido, dentro de su programa de innovación educativa "Aprendizaje y Docencia" $(A+D)$, la puesta en marcha de Proyectos de Innovación y Mejora Educativa (PIME). Durante el curso 2019/2020 se ha implantado un PIME basado en Aprendizaje Basado en Proyectos (ABP) que reúne a dos asignaturas consecutivas de tercer curso de Ingeniería Civil. En este artículo se muestran los resultados alcanzados en el primer semestre de implantación. Los alumnos han expresado claramente su interés y preferencia por este tipo de metodología. Han demostrado haber adquirido un aprendizaje en profundidad y ser capaces de adquirir conocimientos de forma autónoma, así como transferir estos a la realidad de la profesión mediante el desarrollo de su capacidad de trabajar en equipo y la posibilidad de realizar visitas de campo donde los conocimientos recibidos adquieren un carácter práctico. Además, han desarrollado las competencias esperadas, integrando aspectos académicos con otros sociales y éticos.

Palabras clave: PIME, ABP, competencias especificas, competencias transversales, ingeniería civil, docencia activa, carreteras, geotecnia 


\section{Introducción}

En los últimos años, la Universitat Politècnica de València (UPV) ha promovido dentro de su programa de innovación educativa "Aprendizaje y Docencia" $(\mathrm{A}+\mathrm{D})$ la puesta en marcha de Proyectos de Innovación y Mejora Educativa (PIME). Estos proyectos tienen como objetivo fundamental la mejora de la enseñanza y como consecuencia de ello del aprendizaje. La última convocatoria A+D de la UPV apuesta por impulsar el Aprendizaje Basado en Proyectos (ABP)/Aprendizaje Orientado a Proyectos (AOP). El ABP es un método pedagógico que permite al estudiante el desarrollo de competencias específicas y transversales al estar involucrado en un proyecto orientado a solucionar problemas reales que, en el caso de la ingeniería, obliga a integrar el aprendizaje de varias áreas y materias/asignaturas, superando de este modo el aprendizaje fragmentado. Experiencias anteriores sobre la implantación de ABP como estrategia didáctica, en titulaciones de ingeniería, demuestra que los estudiantes logran un aprendizaje contextualizado y significativo (Herrán and Vega, 2006; Case and Light, 2011). Además, esta metodología se confirma como adecuada para evaluar y capacitar al alumno en todo tipo de competencias, específicas y transversales (De Miguel, 2006; Tenza-Abril et al., 2016).

Entre las fortalezas pedagógicas de esta metodología está el hecho de que contribuye a aumentar la atención del alumno y disminuir la evasión de los estudiantes puesto que les exige una participación continua y activa, al tiempo que se ha observado una mayor motivación (Alba Fernández, Torregrosa Cabanilles and del Rey Tormos, 2015; Eucario et al., 2016).

La implantación de esta estrategia metodológica en diversas titulaciones de ingeniería también ha puesto de relieve algunos inconvenientes, como el hecho de que requiere de los estudiantes un bagaje previo en cuanto a resolución de problemas, y que los grupos muy numerosos dificultan la tarea y el seguimiento del docente (Vadillo et al., 2015).

El aprendizaje basado en proyectos sitúa al estudiante como protagonista de su propio aprendizaje ayudando, además, a que los futuros ingenieros se acerquen a un proyecto real de ingeniería antes de finalizar sus estudios (Case y Light, 2011). Con esta técnica los alumnos trabajan en equipo, simulando entornos profesionales en los que aplicar ciertos conocimientos y habilidades. Por otro lado, por la dinámica de trabajo, los alumnos se ven precisados a manejar diferentes fuentes de información, analizar y sintetizar, resolver problemas complejos que abarcan varias disciplinas y, todo ello, les permite mejorar aspectos como la planificación, organización, toma de decisiones, y desarrollar el pensamiento crítico (De Miguel, 2006).

Con estas premisas, los autores del presente artículo, todos ellos profesores de la Escuela Técnica Superior de Ingeniería de Caminos Canales y Puertos (ETSICCP) de la UPV solicitaron en la convocatoria A+D de 2019 un PIME, para la titulación de Ingeniería Civil, de título: "Acercamiento a la realidad profesional a través de un proyecto de acondicionamiento de carreteras desde la geotecnia hasta el diseño de firmes". Algunos de estos profesores cuentan con experiencia previa por su participación en talleres multidisciplinares para la elaboración de trabajos final de grado (TFG), durante los cursos 2014 a 2018, con alumnos de las titulaciones de Ingeniería Civil e Ingeniería de Obras Públicas, con una orientación similar a la prevista para este PIME. Dichos talleres proporcionaron resultados académicos muy satisfactorios, lo que se reflejó en las excelentes calificaciones de cada trabajo individual. Al mismo tiempo, los alumnos que participaron en los mismos calificaron la experiencia de muy interesante y recomendable (Camacho Torregrosa et al., 2015).

El presente PIME reúne a docentes de los departamentos de Ingeniería e Infraestructura de los Transportes (DIIT) e Ingeniería del Terreno (DIT) de la UPV que imparten clases en tercer curso de la titulación de Grado en Ingeniería Civil (GIC) en las asignaturas: "Geotecnia y Cimientos" (12822) y "Caminos y Aeropuertos" (12827). 
Geotecnia y Cimientos es una de las primeras asignaturas tecnológicas para los alumnos del GIC. En general, a los alumnos les cuesta un enorme esfuerzo adaptarse a la forma de trabajo aplicada que las asignaturas tecnológicas requieren. Por otro lado, la ingeniería geotécnica introduce conceptos novedosos y complejos desde el punto de vista de la interpretación física y matemática, que requieren un importante esfuerzo para su compresión y para la integración de los conceptos científicos aprendidos con anterioridad.

La asignatura de Geotecnia y Cimientos proporciona conocimientos básicos teóricos de la Mecánica del Suelo y de la Mecánica de las Rocas, necesarios para poder comprender el comportamiento del terreno, así como las técnicas y procedimientos de trabajo más habituales en el ámbito de la ingeniería geotécnica. Estos conocimientos son básicos para un buen diseño de carreteras, objetivo sobre el que se fundamenta la asignatura Caminos y Aeropuertos. Esta asignatura abarca los conocimientos básicos y esenciales de la ingeniería de carreteras: ingeniería de tráfico, diseño geométrico de carreteras, drenaje y proyecto de explanadas y firmes.

Durante el desarrollo de estas asignaturas, se ha venido observando desmotivación por parte de una amplia mayoría de los estudiantes. Al elevado número de alumnos en los grupos se une la dificultad de las nuevas asignaturas, su desinterés después de pasar por innumerables pruebas de evaluación convencionales, la consecuencia suele ser malos resultados y/o abandono. Además, se ha comprobado que el alumnado presenta serias dificultades para el trabajo colaborativo, siendo la dinámica generalizada el reparto del trabajo entre los integrantes del grupo. Adicionalmente, se observa cómo los alumnos, que deberían llegar a la asignatura de Caminos y Aeropuertos con determinados conocimientos previos, parecen haberlos olvidado o se ven incapaces de integrar estos conceptos con los nuevos aprendizajes.

Parece razonable pensar que el ABP y la conexión transversal entre ellas podrían potenciar la comprensión e integración de conceptos de ambas disciplinas y su aplicación a la situación de un proyecto de carreteras real y, al mismo tiempo, facilitar el aprendizaje. Por ello, se ha llevado a cabo la implantación de esta estrategia didáctica abarcando inicialmente estas dos asignaturas, por su conexión directa en lo que a sus contenidos se refiere y por la facilidad que supone que ambas cuenten con un número de alumnos similar, ya que ambas son de carácter obligatorio y consecutivas en el mismo curso del plan de estudios.

A continuación, en los siguientes apartados se exponen los objetivos y el planteamiento inicial de este PIME, mientras que en el apartado 4 se presentan los resultados parciales obtenidos una vez comenzada su implantación en el primer cuatrimestre del presente curso 2019/2020.

\section{Objetivos}

Los PIMEs son proyectos cuyo propósito fundamental es promover una docencia de carácter activo, orientada a la mejora de la calidad del aprendizaje de los estudiantes. En consecuencia, por definición, los actores de estos proyectos son dos: profesores y estudiantes.

En el PIME que se plantea en este artículo, esto se trata de materializar en dos direcciones:

1. Con respecto al profesorado, se persigue incentivar el trabajo cooperativo en la innovación educativa, desplazando las usuales prácticas aisladas y en solitario en favor de otras desarrolladas por equipos.

2. Con respecto a los estudiantes, se persigue la mejora del aprendizaje con los siguientes objetivos:

- Propiciar un aprendizaje en profundidad, frente a los aprendizajes superficiales.

- Adquirir aprendizajes dirigidos a la transferencia del conocimiento a la vida profesional.

- Promover el desarrollo de habilidades cognitivas diferentes a las convencionales (basadas en la acumulación de conocimientos y los procedimientos memorísticos).

- Desarrollar, al mismo tiempo que las competencias específicas, otras transversales que permitan integrar aspectos académicos con otros sociales, éticos, etc...

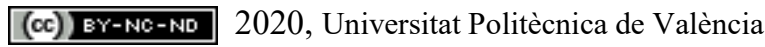

Congreso In-Red (2020) 


\section{Planteamiento inicial del PIME}

El principal objetivo de este proyecto es implementar el aprendizaje basado en proyectos en dos asignaturas del Grado en Ingeniería Civil: Geotecnia y Cimientos y Caminos y Aeropuertos, ambas con una orientación claramente profesional. Para ello, se propuso escoger un tramo real de una carretera convencional y analizar los aspectos geotécnicos (=Geotecnia y Cimientos) que deben ser tenidos en cuenta para mejorar su trazado y adaptarla a las condiciones de trafico actuales (=Caminos y Aeropuertos).

Este trabajo constituye un proyecto real que se incluye en las competencias profesionales del ingeniero civil. De esta forma, los estudiantes se beneficiarían de la estrategia de aprendizaje basado en proyectos, en cada una de las asignaturas, y, además, serían conscientes de la interrelación que existe entre las diferentes asignaturas y de la importancia de no considerarlas como disciplinas estancas.

Este PIME corresponde a la modalidad B: Proyectos de innovación curricular en asignaturas y se ha solicitado con una duración de dos años (2019/2021). Su planteamiento se divide en dos fases: un primer curso piloto, comenzando con las dos asignaturas mencionadas y un segundo curso en el que se tratará de involucrar asignaturas de cuarto curso e incluso se ofrecerá a los alumnos la posibilidad de realizar su TFG continuando con este mismo proyecto. Al tiempo, en el segundo año se tratará de afianzar y mejorar este proyecto en base a la experiencia ya recabada. En la Tabla 1 se recogen el conjunto de tareas previstas en la concepción original del PIME.

Tabla 1. Actividades que constituyen el PIME Ingeniería Geotécnica e Ingeniería de Carreteras

\section{A0 Coordinación}

0.1 Reuniones de seguimiento

0.2 Seguimiento del proyecto por parte de alumnos y profesores

0.3 Consecución de los objetivos

\section{A1 Selección del caso real}

1.1 Definición de criterios para la elección de los tramos de carretera

1.2 Búsqueda y elección de los tramos de carretera

1.3 Preparación del material necesario

1.4 Definición de los proyectos

\section{A2 Selección de alumnos}

\section{A3 Desarrollo de los trabajos}
3.1 Cronograma
3.2 Visitas de campo
3.2 Sesiones grupales
3.3 Entregas

\section{A.4 Competencias transversales}

\section{A.5 Evaluación}

El primer año de implantación el PIME se corresponde con el curso 2019-2020. Ha comenzado en el semestre A con la asignatura Geotecnia y Cimientos, y en semestre actual (semestre B), se está desarrollando en la asignatura Caminos y Aeropuertos. 


\subsection{Selección del caso real}

El trabajo propuesto es el acondicionamiento de una carretera real que presenta deficiencias en cuanto a trazado y adaptación a la normativa vigente en lo que a tráfico se refiere.

Para ello, los profesores del equipo deben realizar la búsqueda y elección de la zona de trabajo en base a los siguientes criterios:

- Carretera convencional de montaña, cuyo trazado se preste a su análisis y rediseño, y pueda ser dividida en varios tramos.

- Datos de tráfico disponibles, que permita realizar un análisis completo del tráfico y de su nivel de servicio.

- Zona donde la variabilidad en la tipología o características del terreno condicionen y/o influyan en el trazado de la carretera.

- Disponibilidad de datos sobre la caracterización del terreno, para poder abordar un análisis geotécnico completo.

Una vez seleccionada la zona y dividida en tramos homogéneos, tantos como grupos de participantes, la información disponible debe ser adaptada a los objetivos que los alumnos deben desarrollar, de manera que conozcan claramente lo que se espera de ellos en cada una de las asignaturas.

\subsection{Selección de alumnos}

La primera fase del PIME consiste en una prueba piloto sobre un grupo reducido de alumnos que voluntariamente quieran participar en el proyecto. La idea inicial era organizar cuatro grupos de trabajo constituidos por cuatro estudiantes cada uno de ellos, por lo que, teniendo en cuenta que tanto la asignatura Geotecnia y Cimientos como la de Caminos y Aeropuertos normalmente cuentan con una matrícula más elevada, es necesaria una selección que debe realizarse en las primeras semanas de clase, coincidiendo con el inicio de la docencia de la primera de estas asignaturas.

Los criterios de selección previstos estarán relacionados con:

- La matrícula de los alumnos. Los estudiantes que participen en el proyecto deben estar matriculados en ambas asignaturas y, preferiblemente (aunque no será condición excluyente), debe ser su primera matrícula.

- El interés de los alumnos, tanto en la participación del proyecto como en continuar con él hasta llegar a realizar su TFG sobre el tema que se desarrolla.

- Si fuera necesario, los resultados en el primer acto de evaluación de la asignatura Geotecnia y Cimientos constituido por una serie de pruebas del minuto que se realizan durante las tres primeras semanas de clase.

En el segundo año de implantación se tratará de ampliar el número de participantes. El número de alumnos definitivo dependerá de los resultados del primer año.

\subsection{Desarrollo de los trabajos}

Las tareas a realizar por los alumnos deben abarcar los contenidos previamente especificados de ambas asignaturas. Estos contenidos se distribuyen en varios ítems que se irán desarrollando en un aula (reservada para este propósito) en formato de taller, en sesiones grupales de dos horas semanales. En este aula los alumnos pueden disponer de un lugar donde trabajar en grupo y/o plantear dudas a los profesores, presentes durante estas sesiones.

(c)) BY-NC-ND 2020, Universitat Politècnica de València

Congreso In-Red (2020) 
Las fechas y horario de estas sesiones deben ser acordadas con los participantes. Para un adecuado seguimiento por parte de los alumnos y de los profesores, tanto la secuncia de actividades como el calendario deben recogerse en un cronograma, que incluya además las fechas de presentación de los diferentes entregables desarrollados por los alumnos.

\subsection{Competencias transversales}

Dentro del plan de estudios de la titulación de Ingeniería Civil, la asignatura Caminos y Aeropuertos es punto de control en la evaluación de dos competencias transversales definidas por la UPV, CT-06: Trabajo en equipo y liderazgo y CT-08: Comunicación efectiva. Ambas competencias se evalúan en el transcurso de la asignatura y, sin duda, pueden evaluarse en el marco del desarrollo del PIME, puesto que la didáctica de esta metodología obliga a los profesores a un contacto directo y un seguimiento continuado del trabajo de los alumnos, que permitirá recoger suficientes evidencias para la evaluación de ambas competencias.

La asignatura Geotecnia y Cimientos es punto de control de las competencias transversales CT-01: Compresión e integración y CT-02: Aplicación y pensamiento práctico, ambas muy necesarias en el planteamiento y conceptualización del problema que los alumnos deben abordar en este proyecto mediante la metodología ABP y fácilmente evaluables mediante el seguimiento continuado de los profesores y las entregas parciales del trabajo que deben realizar.

\subsection{Evaluación del proyecto}

Para un adecuado seguimiento del proyecto se proponen para cada uno de los objetivos específicos una serie de indicadores, que permitan seguir su evolución.

\section{O1 Coordinación de los profesores}

Para una adecuada coordinación de los profesores, es necesario realizar reuniones del grupo. Se consideran dos tipos de reuniones: reuniones de asignatura y reuniones de proyecto. A las reuniones de proyecto deben asistir todos los profesores del grupo, mientras que a las reuniones de asignatura únicamente asistirán los profesores de la propia asignatura y los del grupo que se consideren necesarios.

Indicadores:

- número de reuniones

- asistencia a las mismas

\section{O2 Selección de tramos}

Los tramos objeto de estudio son la base esencial de este proyecto, deben tener las características idóneas para poder desarrollar los objetivos de las dos asignaturas, por lo que no cualquier tramo de carretera sirve.

Indicadores:

- $\quad$ número de tramos evaluados

- número de tramos seleccionados

\section{O3 Selección de los alumnos}

Para comprobar que los criterios de selección de los alumnos han sido adecuados, se analizará cuántos han terminado el proyecto, cuántos han aprobado la asignatura y qué nota han obtenido.

Indicadores:

- número de alumnos que finalizan el proyecto

- número de alumnos que aprueban las dos asignaturas

- nota de los alumnos

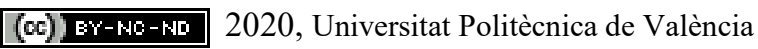

Congreso In-Red (2020) 


\section{O4 Planificación de entregas}

Se realizará un seguimiento de las entregas, tanto de los alumnos como de los profesores.

Indicadores:

- número de entregas de alumnos en tiempo y forma

- número de entregas de las correcciones de los profesores en tiempo y forma

\section{O5 Tutorías}

Se realizará un seguimiento de la asistencia a las tutorías.

Indicadores:

- número de profesores asistentes a cada tutoría

- número de alumnos asistente a cada tutoría

\section{O6 Evolución posterior de los alumnos}

Se realizará un seguimiento de la evolución de los alumnos el curso siguiente al de su matriculación en las asignaturas de Geotecnia y Cimientos y Caminos y Aeropuertos con el fin de comprobar cómo ha influido su participación en el proyecto en su elección de asignaturas en el curso siguiente.

Indicadores:

- número de alumnos matriculados en asignaturas de cuarto curso relacionadas con las del PIME, tales como BIM, Seguridad Vial y Diseño Geotécnico

- número de alumnos que el curso siguiente siguen con el proyecto para su TFG

\section{Resultados}

En el momento de redactar este artículo, el PIME encara el segundo semestre del primer año. Por lo tanto, se presentarán los resultados alcanzados en el primer semestre de implantación. Siguiendo las directrices expuestas en el apartado anterior, el PIME ha sido ya implementado en la asignatura de Geotecnia y Cimientos y se valorarán aspectos como el interés creado en los alumnos, la participación de los mismos, grado de implicación, adaptaciones que han sido necesarias sobre el planteamiento original descrito en el apartado anterior, y resultados académicos alcanzados en la evaluación de esta asignatura, del grupo PIME y en comparación con los resultados obtenidos por el resto de alumnos matriculados en la misma (no adscritos al PIME), que han seguido la metodología tradicional basada en clases magistrales, con resolución de ejercicios prácticos preparados ad hoc para aplicar los conceptos y con un sistema de evaluación constituido por dos pruebas escritas y cuatro pruebas del minuto.

\subsection{Definición de los proyectos}

Tras las primeras reuniones de coordinación se estudiaron varios tramos de carreteras en áreas geográficas distintas y, en función de los datos disponibles, las opciones se redujeron a dos. De estas opciones finales, se eligió el área geográfica con tramos de carreteras que disponían más cantidad de datos técnicos.

En la ubicación elegida se definieron cuatro tramos, uno para cada uno de los cuatro grupos de participantes. De estos cuatro tramos, tres de ellos son bastante homogéneos, salvo el denominado tramo III, ofrece buenas condiciones desde el punto de vista geotécnico y de trazado pero menos en lo que se refiere a la ingeniería de tráfico (Fig. 1). En cualquier caso, todos ellos permiten un desarrollo adecuado de los contenidos de las dos asignaturas implicadas y también de asignaturas de cuarto curso o para que los alumnos puedan realizar su TFG. 


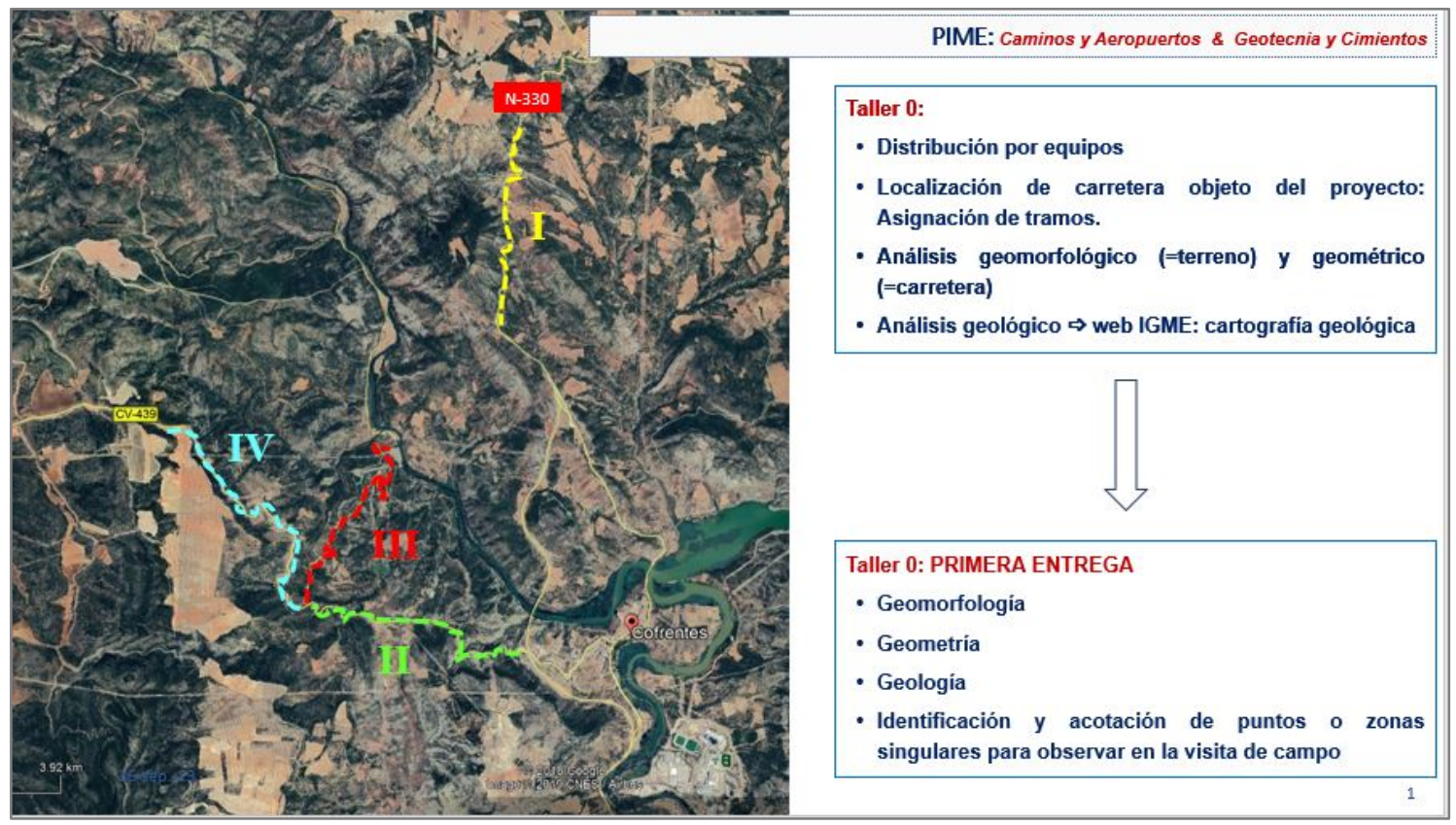

Fig. 1 Diapositiva correspondiente al Taller 0. Muestra la localización de los cuatro subtramos de carretera definidos además de las tareas y contenidos de la primera entrega prevista.

\subsection{Selección de estudiantes}

La primera semana lectiva se envió un correo electrónico a los alumnos matriculados en ambas asignaturas durante el curso 2019/2020, presentando el proyecto y convocándoles a una reunión de presentación del mismo. A esta reunión acudieron 17 alumnos de los 37 posibles candidatos.

El contenido de esta convocatoria era explicar el objetivo, metodología y estructura del PIME, así como las normas de funcionamiento, tales como la obligatoriedad de asistencia a los talleres semanales, compromiso durante todo el proceso, criterios de selección, composición de grupos, actividades previstas, visitas de campo, actos de evaluación, etc...

En el plazo de una semana, 19 alumnos solicitaron participar en el PIME, aunque por motivos no relacionados con la asignatura uno de los alumnos decidió retirarse antes del comienzo del trabajo.

Aunque inicialmente se había fijado el número máximo de 16 participantes (con el fin de distribuirlos en cuatro grupos de cuatro estudiantes), puesto que todos los que lo solicitaron mostraron un elevado interés por participar en el proyecto, el grupo de profesores en la reunión de coordinación decidió admitir a todos ellos. Esto obligó a configurar dos grupos de cinco estudiantes y dos grupos de cuatro estudiantes.

Tabla 2. Composición de los subgrupos de trabajo del PIME

\begin{tabular}{c|c|c|c|c|c}
\hline \multirow{2}{*}{ SUBGRUPO } & ALUMNOS & $\mathbf{1}^{\mathbf{a}}$ MATRÍCULA & \multicolumn{2}{|c|}{ SEXO } & ERASMUS \\
& $\boldsymbol{N}^{\boldsymbol{o}}$ & $\boldsymbol{N}^{\boldsymbol{o}}$ & $\boldsymbol{H}$ & $\boldsymbol{M}$ & $\boldsymbol{N}^{\boldsymbol{j}}$ \\
\hline I & 4 & 4 & 4 & 0 & 0 \\
\hline II & 5 & 5 & 5 & 0 & 0 \\
\hline III & 4 & 3 & 2 & 2 & 1 \\
\hline IV & 5 & 5 & 3 & 2 & 0 \\
\hline Total PIME & $\mathbf{1 8}$ & $\boldsymbol{1 7}$ & $\boldsymbol{1 4}$ & 4 & $\boldsymbol{1}$ \\
\hline
\end{tabular}

(c) 2020 , Universitat Politècnica de València

Congreso In-Red (2020) 
La distribución de los estudiantes en cada grupo fue decidida por ellos mismos, en función de sus preferencias personales y de su grado de afinidad, y la adjudicación de los distintos tramos de carretera se hizo al azar, denominando a cada grupo de trabajo con el número del tramo que se le adjudicó.

Solo uno de los participantes es alumno de segunda matrícula, siendo mayoritariamente chicos. Únicamente cuatro chicas han participado en este PIME y de entre ellas una es estudiante ERASMUS (Tabla 2).

Los subgrupos II y IV están constituidos por alumnos que se conocen desde primer curso de la titulación, habitualmente estudian juntos y se unen para realizar trabajos en grupo. El subgrupo I se conocen y alguna vez han trabajado juntos en asignaturas anteriores. El subgrupo III está constituido por alumnos que no se conocen entre sí y nunca antes han trabajo en el mismo grupo.

Tabla 2. Distribución de alumnos en la asignatura Geotencia y Cimientos

\begin{tabular}{c|c:c|c|c|c|c|c|c|c}
\hline \multirow{2}{*}{ GRUPO } & \multicolumn{2}{|c|}{ ALUMNOS } & \multicolumn{2}{|c|}{$\mathbf{1}^{\mathrm{a} M A T R I ́ C U L A}$} & \multicolumn{3}{|c|}{ SEXO } & \multicolumn{2}{c}{ ERASMUS } \\
& $\boldsymbol{N}^{\boldsymbol{o}}$ & $\mathbf{\%}$ & $\boldsymbol{N}^{\boldsymbol{o}}$ & $\mathbf{\%}$ & $\boldsymbol{H}$ & $\mathbf{\%}$ & $\boldsymbol{M}$ & $\mathbf{\%}$ & $\boldsymbol{N}^{\boldsymbol{o}}$ \\
\hline Total Alumnos matriculados & 89 & $\mathbf{1 0 0}$ & 65 & $\mathbf{7 3 , 0}$ & 47 & $\mathbf{5 2 , 8}$ & 42 & $\mathbf{4 7 , 2}$ & 9 \\
\hdashline Alumnos exceptuando PIME & 71 & $\mathbf{7 9 , 8}$ & 48 & $\mathbf{6 7 , 6}$ & 33 & $\mathbf{4 6 , 5}$ & 38 & $\mathbf{5 3 , 5}$ & 8 \\
\hdashline Participantes PIME & 18 & $\mathbf{2 0 , 2}$ & 17 & $\mathbf{9 4 , 4}$ & 14 & $\mathbf{7 7 , 8}$ & 4 & $\mathbf{2 2 , 2}$ & 1 \\
\hline
\end{tabular}

La Tabla 4 muestra que:

- Los alumnos de primera matrícula, en el conjunto de la asignatura Geotecnia y Cimientos (sin contabilizar los alumnos participantes en el grupo PIME) ha sido del $67.6 \%$ frente al $94.4 \%$ del grupo PIME.

- Casi se da paridad entre alumnos, $52.8 \%$, y alumnas, 47,2 \%. En el grupo PIME la presencia de alumnos es mucho mayor que la de alumnas: $77.8 \%$ frente a $22.2 \%$.

- En la asignatura se han matriculado 9 estudiantes ERASMUS, lo que supone el diez por ciento del total, mientras que solo uno de ellos ha solicitado participar en el PIME.

\subsection{Desarrollo del PIME: Primer año, Semestre A}

Durante el semestre A, el grupo PIME empezó a trabajar en la asignatura Geotecnia y Cimientos. Los alumnos participantes han cursado dos tercios de la asignatura al mismo ritmo y con la misma metodología que el resto de los estudiantes matriculados. Solo en un tercio de la asignatura han seguido el procedimiento $\mathrm{ABP}$ previsto en este proyecto.

Esta decisión se tomó en base a dos consideraciones importantes: la primera de ellas es que para la resolución de problemas reales se considera estrictamente necesario dominar la base de la mecánica del suelo que constituye dos tercios de esta asignatura y, en segundo lugar, poder establecer una comparación entre la metodología convencional mediante clases magistrales y la metodología ABP en el mismo grupo de alumnos.

Se han convocado talleres semanales durante siete semanas de las trece semanas lectivas con que cuenta el calendario oficial para el semestre A de este curso (Fig. 2). Estos talleres se han impartido fuera del horario habitual de las clases de la asignatura y han sustituido a estas. Mientras que no se exige asistencia a las clases magistrales para el resto de alumnos matriculados, la asistencia a los talleres ha sido obligatoria. 


\begin{tabular}{|c|c|c|c|c|c|c|c|c|c|c|c|c|c|}
\hline \multirow{2}{*}{ CURSO 2019-2020 } & \multicolumn{13}{|c|}{ Semestre A } \\
\hline & S1 & $\mathrm{S} 2$ & S3 & S4 & S5 & S6 & S7 & S8 & S9 & $\mathrm{S} 10$ & $\mathrm{~S} 11$ & $\mathrm{~S} 12$ & S13 \\
\hline \multicolumn{14}{|l|}{ Tareas previas: } \\
\hline \multicolumn{14}{|l|}{ 1. Definición del trabajo } \\
\hline \multicolumn{14}{|l|}{ 2. Presentación del PIME } \\
\hline \multicolumn{14}{|l|}{ 3. Selección de alumnos } \\
\hline \multicolumn{14}{|l|}{ Geotecnia y Cimientos } \\
\hline \multicolumn{14}{|l|}{ Visita a la zona de trabajo } \\
\hline \multicolumn{14}{|l|}{ Taller 0: Estudios previos } \\
\hline \multicolumn{14}{|l|}{ Taller 1. Reconocimiento terreno } \\
\hline \multicolumn{14}{|l|}{ Taller 2. Estabilidad de taludes } \\
\hline \multicolumn{14}{|l|}{ Taller 3. Diseño de muros } \\
\hline \multicolumn{14}{|l|}{ Taller 4. Diseño de cimentaciones } \\
\hline Entrega final & & & & & & & & & & & & & \\
\hline
\end{tabular}

Fig. 2 Cronograma propuesto para las actividades a realizar cada una de las semanas del semestre A

Todos los participantes en el PIME han asistido regularmente a las clases magistrales correspondientes a los dos primeros tercios de la asignatura y a los talleres organizados específicamente para este grupo. En cambio, la asistencia regular a las clases magistrales de sus compañeros es del $80 \%$ para el primer tercio de la asignatura, del $50 \%$ al segundo tercio e inferior al $40 \%$ en la recta final del curso.

El cronograma propuesto inicialmente se ha cumplido casi en su totalidad, con solo dos retrasos, la visita de campo debido a las malas condiciones meteorológicas y el Taller 4 retrasado dos semanas por falta de disponibilidad de los profesores, lo que obligó al tiempo a retrasar la entrega final.

Los talleres se han configurado de la siguiente forma:

- Cada taller trata sobre un tema concreto del programa previsto: reconocimiento del terreno, estabilidad de taludes, cimentaciones de estructuras....

- Los primeros minutos se emplean en explicar las bases mínimas necesarias para abordar este tema en el proyecto real de la carretera.

- A continuación, los alumnos distribuidos por grupos resuelven los problemas que presenta a este respecto el subtramo que están estudiando. Los profesores van atendiendo las dudas particulares que van surgiendo a cada grupo, sobre su caso concreto, aprovechando la oportunidad para ampliar los conocimientos teóricos sobre la unidad didáctica que se esté trabajando.

- Los miembros de cada grupo consensuan la forma en que se distribuyen las tareas que deben realizar y van redactando una memoria con las soluciones que van adoptando en cada caso.

- Al finalizar el taller cada grupo entrega el documento que recoge el resultado de su trabajo y un listado con un coeficiente de ponderación del esfuerzo realizado por cada miembro del grupo, a modo de autoevaluación, que deben consensuar entre todos y que debe ser distinto para cada uno.

Una vez finalizado el taller, cada grupo tiene la oportunidad de ampliar y mejorar el trabajo que han entregado con la condición de que el documento definitivo debe ser entregado al inicio del siguiente taller y que deben actualizarse los coeficientes de ponderación del esfuerzo.

Todos los alumnos inscritos en el grupo PIME han participado activamente en los talleres y en el resto de actividades organizadas como las prácticas informáticas o la visita a la zona de estudio, a pesar de realizarlas fuera del horario lectivo (Fig. 3). 


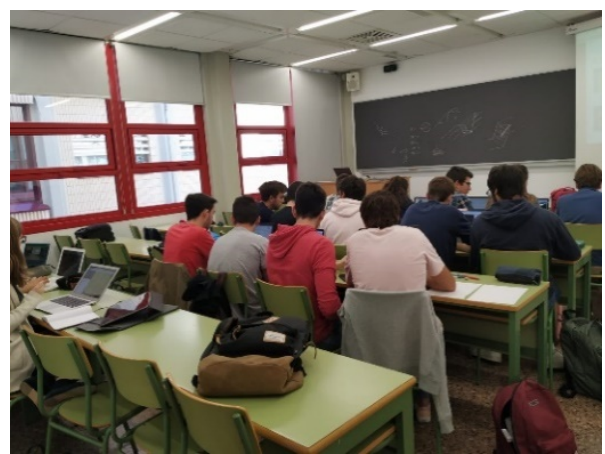

a) Trabajo de grupo en los talleres.

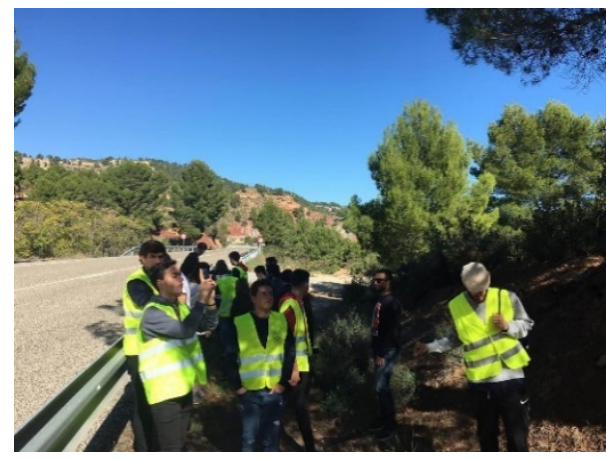

b) Visita a la zona en estudio

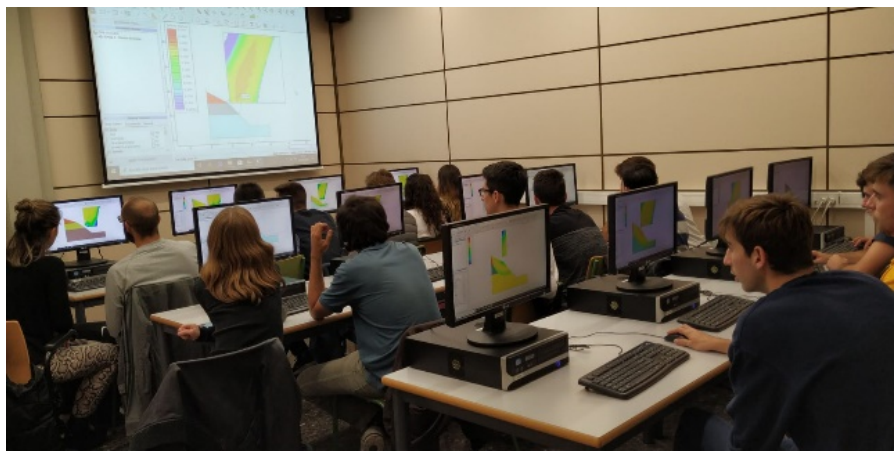

b) Uso de herramientas informáticas especificas

Fig. 3 Distintos tipos de tareas realizadas por los participantes en el PIME

En general, ha sido sencillo para ellos la distribución de las tareas y la asignación de los coeficientes de ponderación del esfuerzo realizado por cada miembro, poniendo en juego competencias distintas de las puramente académicas, demostrando una alta capacidad de trabajo en equipo.

Todos los grupos han mejorado las entregas parciales de cada taller, realizando un trabajo extra fuera del horario establecido, consiguiendo una alta calidad en el trabajo desde el punto de vista técnico y formal.

La dinámica de trabajo, durante los talleres, ha permitido evaluar las competencias transversales de la asignatura Geotecnia y Cimientos de una forma más práctica e individual.

\subsection{Resultados académicos en la asignatura de Geotecnia y Cimientos}

Se ha realizado un estudio comparativo de los resultados académicos alcanzados en los alumnos participantes en el PIME con respecto a los obtenidos por los alumnos que no han participado en el mismo.

Durante este curso los alumnos que no han seguido la metodología $\mathrm{ABP}$ han obtenido resultados similares a los que se vienen alcanzando en curso anteriores. La asignatura está dividida en tres bloques que se evaluan mediante pruebas del minuto (Bloque I) y dos pruebas escritas (Bloques II y III). Generalmente, los alumnos suelen obtener buenos resultados en el primer bloque y estos van disminuyendo a medida que avanza el curso de forma que los peores resultados siempre corresponden al último bloque (Tabla 4). Los alumnos del grupo PIME han roto esta tendencia.

En general, han obtenido resultados similares a los de sus compañeros en los Bloque I y II aunque con una diferencia superior a medio punto sobre aquellos. Estas dos primeras partes de la asignatura han sido comunes para todos los alumnos matriculados. Esto indica que los alumnos que han solicitado participar en el grupo PIME están entre los alumnos más aventajados del curso.

(cc) EY-NC-ND 2020, Universitat Politècnica de València

Congreso In-Red (2020) 
Tabla 3. Resultados académicos

\begin{tabular}{c|c|c|c|c|c}
\hline \multirow{2}{*}{ GRUPO } & \multirow{2}{*}{ DATO } & \multicolumn{4}{|c}{ NOTAS } \\
\cline { 2 - 6 } & & Bloque I & Bloque II & Bloque III & FINAL \\
\hline \multirow{3}{*}{ Subgrupo I } & MEDIA & $\mathbf{8 , 0}$ & $\mathbf{6 , 1}$ & $\mathbf{9 , 0}$ & $\mathbf{7 , 7}$ \\
\cline { 2 - 6 } & DESV_EST & 0,8 & 1,2 & 0,0 & 0,5 \\
\hline \multirow{2}{*}{ Subgrupo II } & MEDIA & $\mathbf{8 , 0}$ & $\mathbf{6 , 0}$ & $\mathbf{1 0 , 0}$ & $\mathbf{8 , 0}$ \\
\cline { 2 - 6 } & DESV_EST & 0,8 & 1,4 & 0,0 & 0,5 \\
\hline \multirow{3}{*}{ Subgrupo III } & MEDIA & $\mathbf{6 , 7}$ & $\mathbf{5 , 4}$ & $\mathbf{7 , 8}$ & $\mathbf{6 , 6}$ \\
\cline { 2 - 6 } & DESV_EST & 0,2 & 1,6 & 2,5 & 1,1 \\
\hline \multirow{3}{*}{ Subgrupo IV } & MEDIA & $\mathbf{8 , 2}$ & $\mathbf{7 , 0}$ & $\mathbf{1 0 , 0}$ & $\mathbf{8 , 4}$ \\
\cline { 2 - 6 } & DESV_EST & 1,5 & 1,3 & 0,0 & 0,8 \\
\hline \multirow{2}{*}{ Total PIME } & MEDIA & $\mathbf{7 , 8}$ & $\mathbf{6 , 2}$ & $\mathbf{9 , 3}$ & $\mathbf{7 , 7}$ \\
\cline { 2 - 6 } & DESV_EST & 1,1 & 1,4 & 1,4 & 1,0 \\
\hline \multirow{2}{*}{ Alumnos excepto PIME } & MEDIA & $\mathbf{7 , 0}$ & $\mathbf{5 , 5}$ & $\mathbf{5 , 3}$ & $\mathbf{5 , 9}$ \\
\cline { 2 - 6 } & DESV_EST & 1,5 & 1,8 & 1,9 & 1,5 \\
\hline \multirow{2}{*}{ Total Alumnos } & MEDIA & $\mathbf{7 , 2}$ & $\mathbf{5 , 6}$ & $\mathbf{6 , 1}$ & $\mathbf{6 , 3}$ \\
\cline { 2 - 6 } & DESV_EST & 1,5 & 1,7 & 2,5 & 1,6 \\
\hline
\end{tabular}

La diferencia más importante se observa en el Bloque III, que corresponde a la parte de la asignatura que se ha trabajado con metodología ABP en el grupo PIME y mediante clases magistrales en el grupo de aula. Los alumnos del PIME han obtenido una nota media de 9.3 frente a 5.3 del resto de sus compañeros.

Dos razones justifican estos resultados: la primera de ellas es que los alumnos participantes en el PIME son alumnos aventajados, que han obtenido mejores resultados en las tres partes de la asignatura; y la segunda razón es la obligatoriedad de asistencia a los talleres y las estrictas fechas de entrega de los resultados que les obliga a mantener sus estudios al día mientras que el resto de los alumnos, en esta recta final de semestre, van dejando de asistir a clase y preparan los examenes con solo unos días de antelación.

El grupo PIME cuenta con la ventaja de la aplicación directa de los conocimientos adquiridos a un proyecto real. Por contra, han contado con una desventaja muy importante, ya que deben preparar casi al mismo tiempo los contenidos de los bloques II y III, de forma que han asistido al mismo tiempo al aula habitual y a los talleres, por tanto, han contado con menos tiempo disponible en la parte central del semestre y han tenido que resolver problemas al mismo tiempo que adquirian las bases teóricas minimas necesarias. Esto se ha reflejado en los resultados obtenidos en la prueba escrita correspondiente al bloque II, que son menores a los que han obtenido en los bloque I y III, a pesar de que son mayores en más de medio punto a los obtenidos por el resto de sus compañeros.

En cuanto a los distintos subgrupos en que se ha distribuido al grupo, se observa que los peores resultados corresponden al subgrupo III formado por alumnos que nunca antes habían trabajado en grupo entre ellos. Al mismo tiempo, los alumnos de este subgrupo son los que han obtenido peores resultados también en las dos primeras partes de la asignatura, estando sus notas medias por debajo de las medias obtenidas por el resto de sus compañeros, tanto de aula como del grupo PIME (Tabla 4, valores en rojo). 


\section{Conclusiones}

Los resultados observados por los profesores y expresados por los alumnos participantes en este PIME avalan las hipotesis de partida, a saber, el ABP es un método pedagógico que permite al estudiante el desarrollo de competencias específicas y transversales, proporciona un aprendizaje contextualizado y significativo, contribuye a aumentar la atención del alumno y disminuir la evasión al exigir una participación continua y activa, al tiempo que aumenta su motivación.

En general, se concluye que:

- Han solicitado participar en el PIME el $50 \%$ del total de alumnos que estaban en condiciones de participar en el mismo por estar matriculados durante el mismo curso en las asignaturas de Geotencia y Cimientos y Caminos y Aeropuertos.

- El desarrollo del PIME en el semestre A del primer año ha demostrado el acierto de las previsiones en lo que a la distribución de actividades y cronograma de las mismas. Por otro lado, ha puesto de manifiesto la necesidad de ajustar agunos contenidos a la realidad de la profesión.

- La selección del caso real ha sido acertada, pues todos los alumnos han contado con suficientes datos técnicos para resolver los condicionantes geotécnicos del subtramo que han estudiado y, a pesar de la cercanía de los tramos, ha habido suficientes diferencias entre ellos para que cada grupo haya ofrecido un resultado distinto.

- La selección de alumnos ha seguido criterios adecuados, dados los resultados obtenidos. Probablemente los alumnos que han participado en el PIME hubiesen obtenido buenos resultados en la asignatura, puesto que son en general alumnos con buenos resultados académicos.

- La distribución de alumnos por grupo ha demostrado que los resultados son mejores cuando los alumnos que conforman un grupo se conocen entre sí y estudian en grupo habitualmente.

- Las visitas a la zona de estudio y el trabajo en grupo permiten transferir de una forma más efectiva los conocimientos aquiridos a la realidad de la práctica profesional del ingeniero civil.

Con el desarrollo de esta primera parte del PIME se puede concluir que el grado de alcance de los objetivos ha sido elevado por parte de los profesores y alumnos.

En lo que se refiere a los profesores, hemos sido concientes de las ventajas de programar de forma coordinada nuestras asignaturas a pesar del esfuerzo extra que supone adaptar los contenidos y la organización de las mismas, dado su carácter consecutivo a lo largo del curso, y a su orientación profesional al trabajar sobre un proyecto real.

En cuanto a los alumnos, éstos han expresado claramente su interes y preferencia por este tipo de metodologia, han demostrado haber adquirido un aprendizaje en profundidad y ser capaces de adquirir otros conocimientos de forma autónoma, así como transferir estos a la realidad de la profesión. Han adquirido habilidades cognitivas diferentes y desarrollado las competencias esperadas, integrando los aspectos académicos con otros sociales y éticos.

\section{Referencias}

ALBA FERNÁNDEZ, J., TORREGROSA CABANILLES, C. AND DEL REY TORMOS, R. (2015) 'Aprendizaje basado en proyectos: Primera experiencia en la asignatura de Física del Grado en Ingeniería de Telecomunicación, Sonido e Imagen', pp. 278-287. doi: 10.4995/inred2015.2015.1591.

CAMACHO TORREGROSA, F. J. et al. (2015) 'Desarrollo de un Trabajo de Fin de Grado Multidisciplinar en Ingeniería Civil como preparación del alumno para la vida profesional', pp. 1249-1263. doi: 10.4995/inred2015.2015.1635.

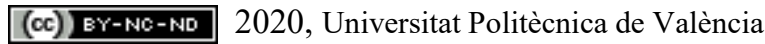

Congreso In-Red (2020) 
CASE, J. M. AND LIGHT, G. (2011) 'Emerging methodologies in engineering education research', Journal of Engineering Education, 100(1), pp. 186-210. doi: 10.1002/j.2168-9830.2011.tb00008.x.

EUCARIO, J. et al. (2016) 'Aprendizaje basado en problemas en el camino a la innovación en ingeniería', 7(2), pp. 966-103.

GARRIDO DE LA TORRE, M. E. et al. (2016).’TFG multidisciplinar en el área de la ingeniería civil” Jornadas Red Estatal de Docencia Universitaria (RED-U 2016). TFG/TFM. (189 - 195). Madrid: Red Estatal de Docencia Universitaria (RED-U).

HERRÁN, C. A. AND VEGA, C. F. (2006) 'Uso del ABP como estrategia didáctica para lograr aprendizaje significativo del diseño de ingeniería', Revista Educación en Ingeniería, 1(2), pp. 33-44. Available at: http://www.educacioneningenieria.org/index.php/edi/article/view/39.

MARIO DE MIGUEL DÍAZ (2005) Modalidades de enseñanza centradas en el desarrollo de competencias. Orientaciones para promover el cambio metodológico en el marco del EEES. Available at: http://www.uvic.es/sites/default/files/Ensenanza_para_competencias.PDF.

TENZA-ABRIL, A. J. et al. (2016) 'Aprendizaje basado en proyectos en la asignatura Técnicas de Investigación en Ingeniería Geológica', XIV Jornadas de Redes de Investigación en Docencia Universitaria. Investigación, innovación $y$ enseñanza universitaria: enfoques pluridisciplinares, pp. 2314-2326. Available at: http://rua.ua.es/dspace/handle/10045/59694.

VADILLO, J. Á. et al. (2015) 'Análisis de los resultados de la implantación ABP en un Grado de Ingeniería Informática', Actas del simposio-taller sobre estrategias y herramientas para el aprendizaje y la evaluación, pp. 2-9. 